\title{
What drives depression in empty nose syndrome? A Sinonasal Outcome Test-25 subdomain analysis*
}

\author{
Chien-Chia Huang ${ }^{1,2}$, Pei-Wen Wu ${ }^{1,3}$, Chia-Hsiang Fu' ${ }^{1,2}$, Chi-Che Huang ${ }^{1,2}$, \\ Po-Hung Chang ${ }^{1,2}$, Ching-Lung Wu', Ta-Jen Lee ${ }^{1,4}$ \\ ' Division of Rhinology, Department of Otolaryngology, Chang Gung Memorial Hospital and Chang Gung University, Taoyuan, \\ Taiwan \\ ${ }^{2}$ Graduate Institute of Clinical Medical Sciences, College of Medicine, Chang Gung University, Taiwan \\ ${ }^{3}$ Department of Otolaryngology-Head and Neck Surgery, Chang Gung Memorial Hospital and Chang Gung University, Keelung, \\ Taiwan \\ ${ }^{4}$ Department of Otolaryngology, Xiamen Chang Gung Hospital, Xiamen, China
}

Rhinology 57: 6, $469-476,2019$

https://doi.org/10.4193/Rhin19.085

*Received for publication:

February 7, 2019

Accepted: July 12, 2019

\begin{abstract}
Background: Empty nose syndrome (ENS) is a debilitating disorder characterised by paradoxical nasal obstruction after excessive surgical excision of nasal tissues. ENS negatively impacts the quality of life (QOL) and psychological status of patients.

This study aimed to determine the associations among disease-specific QOL impairments and the severity of anxiety and depression before and after surgery in ENS patients.
\end{abstract}

Methods: A total of 68 ENS patients were prospectively recruited and underwent submucosal Medpor implantation. QOL impairments and the severity of anxiety and depression were evaluated using the Sinonasal Outcome Test-25 (SNOT-25), Beck Depression Inventory-II (BDI-II), and Beck Anxiety Inventory (BAI) 1 day before and 6 months after surgery.

Results: The BDI-II and BAI scores were significantly associated with the total score and ear/facial symptoms, psychological dysfunction, sleep dysfunction, and empty nose symptoms domains of the SNOT-25. Surgery improved disease-specific and psychological symptoms. Post-operative changes in the BDI-II score were correlated with changes in the total score and sleep dysfunction and empty nose symptoms domains of the SNOT-25. A SNOT-25 total score of $>60$, sleep dysfunction domain score of $>18$, and empty nose symptoms domain score of $>14$ were good predictors of moderate-to-severe depression.

Conclusions: ENS symptoms are associated with psychological burden and could be good predictors of moderate-to-severe depression. Targeted symptom improvement could reduce the psychological burden.

Key words: beck anxiety inventory, beck depression inventory II, depression, empty nose syndrome, Sinonasal Outcome Test-25

\section{Introduction}

Empty nose syndrome (ENS) is a debilitating disorder reported to arise as a post-surgical paradoxical nasal obstruction from excessive excision of nasal tissues ${ }^{(1)}$. These patients suffer from subjective nasal stuffiness despite the large air space observed in the nasal cavity ${ }^{(2)}$. The pathophysiology of this paradoxical phenomenon is complicated and not yet fully understood. ENS is also reported in persons with normal turbinate and intranasal volume, and not every patient undergoing a radical turbinate procedure experiences the symptoms of $\mathrm{ENS}^{(3)}$. Abnormalities in neurosensory systems due to improper mucosal healing and al- terations to the nasal airflow after surgical insult are proposed to play major roles in the abnormal sensations of ENS patients ${ }^{(3-6)}$. Patients with ENS may also experience mucosal dryness, nasal congestion, facial pain and headache on inspiration, and excessive nasal crusting and discharge ${ }^{(1,2,7)}$. Affected patients often report a significant negative impact on the quality of life (QOL) and psychological status, productivity loss, and lifestyle disruption $^{(8-10)}$. As a result, a validated instrument, the Sinonasal Outcome Test-25 (SNOT-25), was developed by Houser ${ }^{(2)}$. The SNOT-25 is a modification of the SNOT-20 that measures five additional symptoms: dryness, difficulty with nasal breathing, suffocation, 
excessively open nose, and nasal crusting. These five additional items may better assess the symptoms of ENS patients $s^{(7)}$. ENS patients also experience anxiety and depression that may lead to suicide attempts in extreme $\operatorname{cases}^{(8-10)}$. Our previous study ${ }^{(10)}$ using validated instruments including the Beck Depression Inventory-II (BDI-II) ${ }^{(11)}$ and Beck Anxiety Inventory (BAI) ${ }^{(12)}$ found that depression and anxiety are common among ENS patients and could be effectively improved after surgical treatment. Furthermore, submucosal implantation with porous high density polyethylene (Medpor; Porex Surgical, Inc.) provided better outcomes when implanted in the lateral nasal wall than in the inferior nasal wall ${ }^{(13)}$. Given this association, it is likely that impairments in each of the QOL domains within the SNOT-25 would differentially affect the severity of anxiety and depression. As such, the aim of this study was to evaluate the associations of each SNOT-25 subdomain with the severity of anxiety and depression before and after surgical treatment in patients with ENS. Differentiating the QOL domains that are associated with psychological dysfunction may be useful for targeted symptom improvement to reduce psychological burden and optimise patient-centred care.

\section{Materials and methods}

Patients

The study was approved by the institutional review board of the Chang Gung Memorial Hospital (IRB number: 201506433A3 and 201601703A3). We prospectively recruited consecutive patients with ENS who were managed in the department of otolaryngology between 2015 and 2018, and all participants provided signed informed consent prior to enrolment. Patients were diagnosed with ENS based on subjective symptoms such as paradoxical nasal obstruction, breathing discomfort, and other related discomfort, as well as consistent physical examinations including a cotton test. In the cotton test, a moistened cotton ball was placed in the widest area of the common nasal cavity in which previous procedures were performed. Improvement in symptoms as the patient was requested to breathe through the nose for 30 min was indicative of a positive cotton test ${ }^{(2)}$. The patients' demographic data and clinical characteristics were recorded. Nasal endoscopy was performed to evaluate the nasal cavity before and after treatment in all patients. The exclusion criteria included patients with congenital craniofacial anomaly or other sinonasal diseases such as rhinosinusitis or nasal polyps, and patients that had been diagnosed with psychiatric disorders including schizophrenia, bipolar disorder, and major depression by the psychiatrists or had received antipsychotics prior to the initial nasal surgeries.

\section{Surgical intervention}

Patients were surgically treated by the same surgeon (Ta-Jen Lee) with endoscopic-assisted submucosal Medpor implanta-
Table 1. Categorized survey items for separate domains of the SNOT-25 questionnaire.

\begin{tabular}{|lll|}
\hline SNOT-25 domains & Items & $\begin{array}{l}\text { Score } \\
\text { range }\end{array}$ \\
\hline Rhinologic symptoms & $1,2,3,6,22$ & $0-25$ \\
\hline Extra-nasal rhinologic symptoms & $4,5,6$ & $0-15$ \\
\hline Ear/facial symptoms & $2,7,8,9,10$ & $0-25$ \\
\hline Sleep dysfunction & $11,12,13,14,15$ & $0-25$ \\
\hline Psychological dysfunction & $14,15,16,17,18,19,20$ & $0-35$ \\
\hline Empty nose symptoms & $21,22,23,24,25$ & $0-25$ \\
\hline
\end{tabular}

SNOT-25, Sino-Nasal Outcome Test-25.

tion, as described previously ${ }^{(10,13,14)}$. In brief, under local anaesthesia, an incision was made on the nasal floor or lateral wall to create a submucosal pocket for the Medpor implantation. The Medpor implant, a nonreactive material that allows tissue and vascular ingrowth, was sized between $8 \times 25 \mathrm{~mm}$ and $8 \times 40 \mathrm{~mm}$ and inserted. The volume of the Medpor graft used to form an ideal implant contour was based on the length of the patient's nasal floor or inferior meatus. It was crucial to avoid injury to the valve of Hasner, maintain the integrity of the mucosal flap, and prevent protrusion of the implants.

Patient-reported outcome and psychological status measures

Patients completed surveys 1 day before and 6 months after surgical treatment. The assessment of QOL was performed using the SNOT-25, which is scored from 0 (no symptoms) to 5 (severe symptoms) for each item. For analysis, the SNOT-25 questionnaire was categorised into six domains according to a previous study ${ }^{(7)}$ : rhinogenic symptoms, extra-nasal rhinological symptoms, ear/facial symptoms, sleep dysfunction, psychological dysfunction, and empty nose symptoms (Table 1).

The psychological status of ENS patients was evaluated using the Chinese versions of the BDI-II and BAI pre- and 6 months post-operatively ${ }^{(15)}$. The BDI-II and BAI are each composed of 21 items scored between 0 (symptom not present) and 3 (symptom strongly present) with resulting total scores of $0-63$. A total BDI-II score of 0-13 indicates normal, 14-19 indicates mild depression, 20-28 indicates moderate depression, and 29-63 indicates severe depression ${ }^{(11,16)}$. Similarly, a total BAI score of 0-7 indicates normal, 8-15 indicates mild anxiety, 16-25 indicates moderate anxiety, and 26-63 indicates severe anxiety ${ }^{(12)}$.

\section{Statistical analyses}

The data are presented as the mean \pm standard deviation and were statistically analysed using GraphPad Prism 5 (GraphPad Prism Software, Inc, San Diego, Calif). Categorical variables were 
Table S. Clinical characteristics of the study participants (pre-operative).

\begin{tabular}{|c|c|c|c|c|}
\hline & & Post-op & months & \\
\hline & Total & Complete & Incomplete & P value $^{+}$ \\
\hline Case number & 68 & 39 & 29 & \\
\hline Age & $50.4 \pm 12.6$ & $51.1 \pm 11.6$ & $49.5 \pm 12.7$ & ns \\
\hline Female : male (n) & $17: 51$ & $14: 25$ & $3: 26$ & $0.016^{*}$ \\
\hline Smoker (n, \%) & 9 & 5 & 4 & ns \\
\hline Serum lgE (IU/ml) & $323.7 \pm 972.3$ & $138.8 \pm 141.5$ & $581.2 \pm 1471$ & ns \\
\hline BDI-II score & $19.3 \pm 15.0$ & $21.3 \pm 15.0$ & $16.7 \pm 14.9$ & ns \\
\hline BAl score & $17.7 \pm 12.9$ & $21.2 \pm 13.4$ & $13.0 \pm 10.6$ & $0.007^{* *}$ \\
\hline SNOT-25 total score & $62.9 \pm 25.3$ & $63.3 \pm 22.4$ & $62.4 \pm 29.2$ & ns \\
\hline Rhinologic symptoms & $11.8 \pm 6.4$ & $12.0 \pm 5.6$ & $11.6 \pm 7.5$ & ns \\
\hline Extra-nasal rhinologic symptoms & $6.9 \pm 4.6$ & $6.9 \pm 4.5$ & $7.3 \pm 4.7$ & ns \\
\hline Ear/facial symptoms & $8.4 \pm 5.7$ & $8.7 \pm 5.6$ & $8.0 \pm 6.0$ & ns \\
\hline Sleep dysfunction & $16.7 \pm 7.3$ & $16.9 \pm 6.7$ & $16.5 \pm 8.1$ & ns \\
\hline Psychological dysfunction & $21.6 \pm 10.4$ & $21.5 \pm 10.2$ & $21.6 \pm 10.7$ & ns \\
\hline Empty nose symptoms & $12.9 \pm 5.4$ & $12.6 \pm 5.3$ & $13.4 \pm 5.6$ & ns \\
\hline
\end{tabular}

Data are represented as mean \pm standard deviation. SNOT-25, sino-nasal outcome test-25; BDI-II, Beck Depression Inventory II; BAI, Beck Anxiety Inventory. ${ }^{\dagger}$ Paired $t$ test analysed between pre-operative and 6 months post-operative symptom scores. ${ }^{*} p<0.05,{ }^{* *} p<0.01,{ }^{* * *} p<0.001$.

compared using the $\chi^{2}$ test. Continuous variables were analysed using unpaired or paired $t$ tests between two groups or pre- and post-operative changes, as appropriate. Correlations were determined using Pearson's correlation coefficient $(r, 0-0.2$ : very weak, 0.2-0.4: weak, 0.4-0.6: moderate, 0.6-.0.8: strong, 0.8-1.0: very strong correlation). To identify and characterise the sensitivity and specificity of our metrics for SNOT-25 for the detection of moderate and severe depression in ENS participants, receiver operating characteristic (ROC) curves were analysed and the area under the ROC curve (AUC) was calculated. Statistical significance was set at $\mathrm{p}<0.05$.

\section{Results}

\section{Clinical characteristics of the study population}

A total of 68 ENS patients were enrolled during the study period. The general characteristics of the participants (pre-operative) are summarised in Table $S$. There were no differences in age, cigarette smoking status, serum immunoglobulin (lg)E level, SNOT-25 score, and BDI-II score between the 39 participants with and 29 participants without complete follow-up and evaluation 6 months post-operatively. However, differences were observed in sex and the baseline BAI scores between these participants. According to the statements from participants and findings on nasal endoscopy or sinus computed tomography, all patients had undergone inferior turbinate surgery. Thirty-one (45.6\%) patients underwent nasal surgery more than once. Forty-one (60.3\%) patients underwent simultaneous nasal septal surgery. Twenty-seven (39.7\%) patients underwent concurrent sinus surgery, 23 of whom underwent endoscopic sinus surgery and six of whom underwent Caldwell-Luc operation.

The initial mean baseline BDI-II score was $19.3 \pm 15.0$. Thirtythree (48.5\%) patients had scores of 0-13 (indicating normal), nine (12.2\%) patients had scores of 14-19 (indicating mild depression), five (7.4\%) patients had scores of 20-28 (indicating moderate depression), and 21 (30.9\%) patients had scores of 29-63 (indicating severe depression). The initial mean baseline BAI score was $17.7 \pm 12.9$. Eighteen (26.5\%) patients had scores of 0-7 (indicating normal), 14 (20.6\%) patients had scores of 8-15 (indicating mild anxiety), 18 (26.5\%) patients had score of 16-25 (indicating moderate anxiety), and 18 (26.5\%) patients had scores of 26-63 (indicating severe anxiety).

\section{Associations among the BDI-II, BAI, and SNOT-25 scores}

The BDI-II score was significantly associated with the total score and ear/facial symptoms, psychological dysfunction, sleep dysfunction, and empty nose symptoms domains of the SNOT-25; but not with the rhinogenic symptoms and extra-nasal rhinological symptoms domains of the SNOT-25 (Figure 1). Similarly, the BAI score was significantly associated with the total score and ear/facial symptoms, psychological dysfunction, sleep dysfunction, and empty nose symptoms domains of the SNOT-25; but not with the rhinogenic symptoms and extra-nasal rhinological symptoms domains of the SNOT-25 (Figure 2). In addition, multivariate linear regression analysis was conducted to adjust 

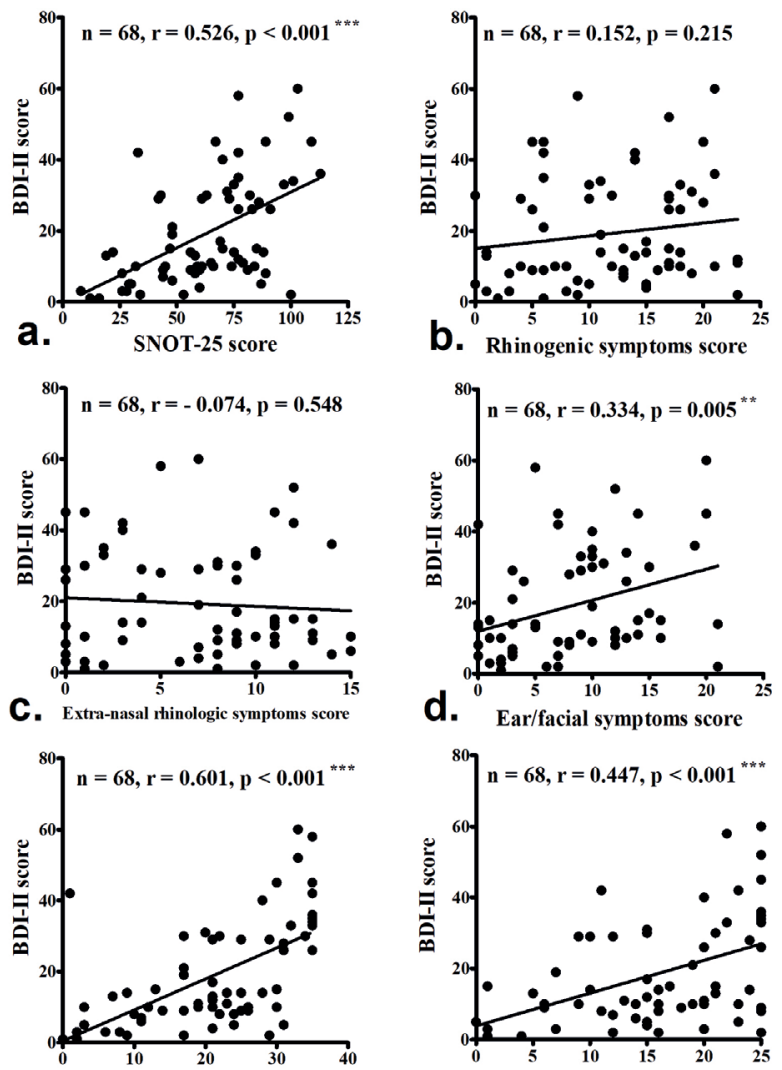

e. Psychological dysfunction score

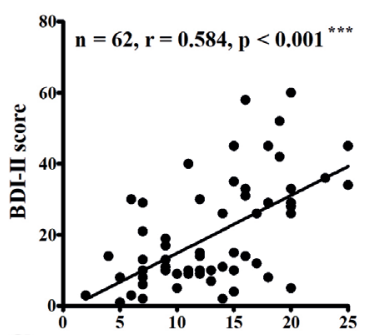

g. ENS symptoms score

Figure 1. The Beck Depression Inventory II (BDI-II) score was significantly associated with the Sinonasal Outcome Test-25 (SNOT-25) total score (a), ear/facial symptoms domain (d), psychological dysfunction domain (e), sleep dysfunction domain ( $f$ ), and empty nose symptoms (ENS) domain (g), but not with the rhinogenic symptoms domain (b) or extra-nasal rhinologic symptoms domain (c). The correlation was determined using Pearson's correlation coefficient ( $r, 0-0.2$ : very weak, $0.2-0.4$ : weak, $0.4-$ 0.6: moderate, $0.6-$-.0.8: strong, $0.8-1.0$ : very strong correlation). ${ }^{*} \mathrm{p}<$ $0.05,{ }^{* *} \mathrm{p}<0.01,{ }^{* * *} \mathrm{p}<0.001$.

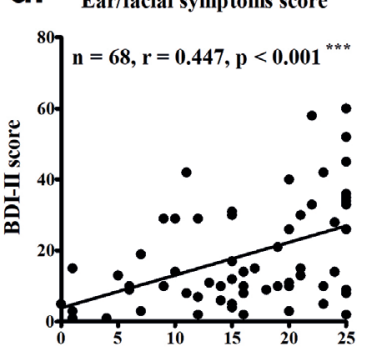

f. Sleep dysfunction score
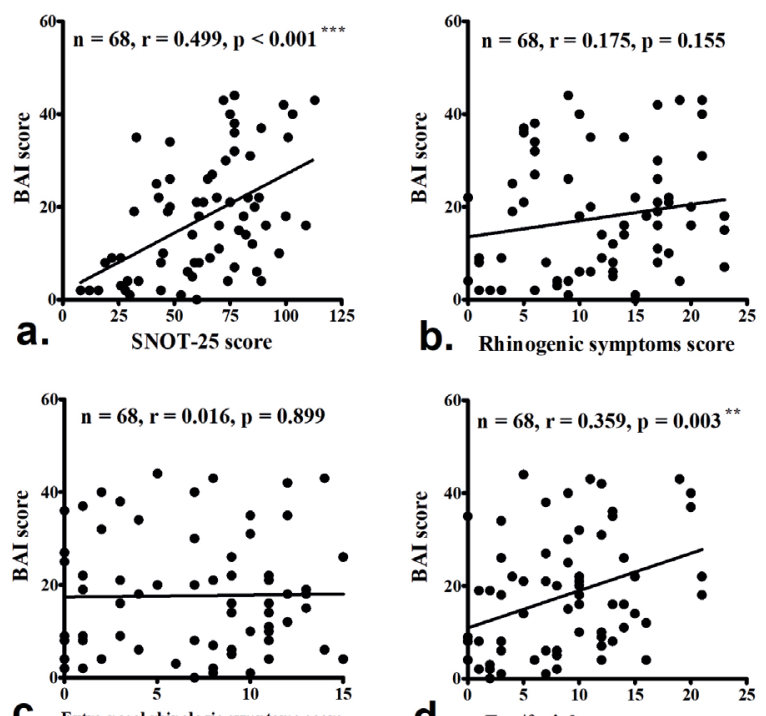

C. Extra-nasal rhinologic symptoms score

d. Ear/facial symptoms score

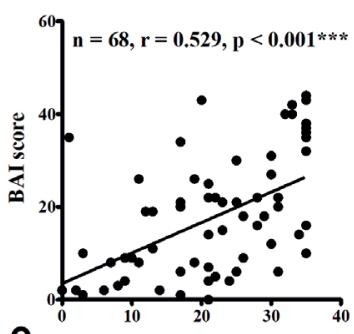

P. Psychological dysfunction score

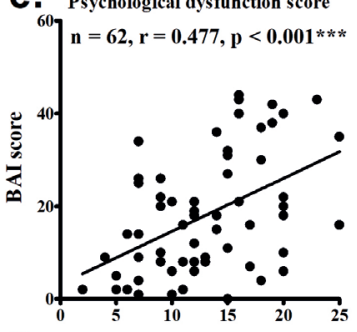

f.

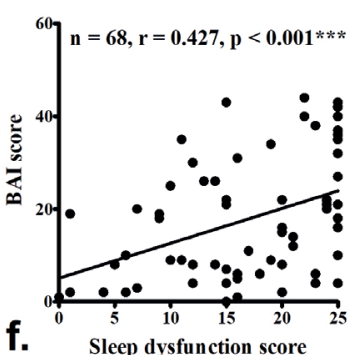

g.

Figure 2. The Beck Anxiety Inventory (BAI) score was significantly associated with the Sinonasal Outcome Test-25 (SNOT-25) total score (a), ear/ facial symptoms domain (d), psychological dysfunction domain (e), sleep dysfunction domain (f), and empty nose symptoms (ENS) domain $(\mathrm{g})$, but not with the rhinogenic symptoms domain (b) or extra-nasal rhinologic symptoms domain (c). The correlation was determined using Pearson's correlation coefficient. ${ }^{*} p<0.05,{ }^{* *} p<0.01,{ }^{* * *} p<0.001$. the age, gender, education level, smoking status, and serum total IgE, after which the pre-operative SNOT-25 total score was found to be associated with the BDI-II and BAI scores (coefficient $=0.305$ and 0.231 , respectively, both $p<0.001$ ).

\section{Clinical outcomes after nasal surgery}

Nasal surgery with Medpor implantation improved most QOL symptoms as measured by the SNOT-25 (with the exception of nasal crusting and facial pain/pressure) and psychological status as measured by the BDI-II and BAI (Table 2). Post-operative changes in the BDI-II score were significantly associated with changes in the total score and sleep dysfunction and empty nose symptoms domains of the SNOT-25; but not with changes in the rhinogenic symptoms, extra-nasal rhinological symptoms, and ear/facial symptoms domains of the SNOT-25 (Figure 3). 
Table 2. The symptom scores reported by participants before and 6 months after surgery.

\begin{tabular}{|c|c|c|c|}
\hline Items & $\begin{array}{l}\text { Pre-opera- } \\
\text { tive }(n=68)\end{array}$ & $\begin{array}{l}\text { Post-opera- } \\
\text { tive }(n=39)\end{array}$ & P valuet \\
\hline 1. Need to blow nose & $2.3 \pm 1.8$ & $1.5 \pm 1.3$ & $0.008^{* *}$ \\
\hline 2. Sneezing & $1.7 \pm 1.4$ & $1.2 \pm 1.1$ & $0.024^{*}$ \\
\hline 3. Runny nose & $1.9 \pm 1.7$ & $1.4 \pm 1.1$ & $0.017^{*}$ \\
\hline 4. Cough & $1.9 \pm 1.6$ & $0.9 \pm 1.0$ & $0.002^{* *}$ \\
\hline 5. Postnasal discharge & $2.7 \pm 2.0$ & $1.4 \pm 1.4$ & $<0.001^{* * *}$ \\
\hline 6. Thick nasal discharge & $2.4 \pm 1.9$ & $1.4 \pm 1.5$ & $0.027^{*}$ \\
\hline 7. Ear fullness & $1.9 \pm 1.7$ & $1.0 \pm 1.5$ & $0.009^{* *}$ \\
\hline 8. Dizziness & $2.3 \pm 1.7$ & $1.2 \pm 1.5$ & $<0.001^{* * *}$ \\
\hline 9. Ear pain & $1.3 \pm 1.5$ & $0.7 \pm 1.1$ & $0.024^{*}$ \\
\hline 10. Facial pain/pressure & $1.3 \pm 1.7$ & $0.8 \pm 1.3$ & 0.146 \\
\hline $\begin{array}{l}\text { 11. Difficulty falling } \\
\text { asleep }\end{array}$ & $3.0 \pm 1.8$ & $1.3 \pm 1.4$ & $<0.001^{* * *}$ \\
\hline 12. Waking up at night & $3.2 \pm 1.7$ & $1.7 \pm 1.5$ & $<0.001^{* * *}$ \\
\hline $\begin{array}{l}\text { 13. Lack of good night's } \\
\text { sleep }\end{array}$ & $3.6 \pm 1.6$ & $1.8 \pm 1.6$ & $<0.001^{* * *}$ \\
\hline 14. Waking up tired & $3.5 \pm 1.6$ & $1.8 \pm 1.5$ & $<0.001^{* * *}$ \\
\hline 15. Fatigue & $3.4 \pm 1.6$ & $1.9 \pm 1.5$ & $<0.001^{* * *}$ \\
\hline $\begin{array}{l}\text { 16. Reduced produc- } \\
\text { tivity }\end{array}$ & $3.1 \pm 1.6$ & $1.6 \pm 1.4$ & $<0.001^{* * *}$ \\
\hline $\begin{array}{l}\text { 17. Reduced concen- } \\
\text { tration }\end{array}$ & $3.2 \pm 1.7$ & $1.6 \pm 1.4$ & $<0.001^{* * *}$ \\
\hline $\begin{array}{l}\text { 18.Frustration/restless- } \\
\text { ness/irritability }\end{array}$ & $3.2 \pm 1.7$ & $1.5 \pm 1.4$ & $<0.001^{* * *}$ \\
\hline 19. Sadness & $2.8 \pm 1.8$ & $1.5 \pm 1.4$ & $<0.001^{* * *}$ \\
\hline 20. Embarrassment & $2.4 \pm 1.9$ & $1.0 \pm 1.2$ & $<0.001^{* * *}$ \\
\hline 21. Dryness & $3.2 \pm 1.6$ & $1.5 \pm 1.3$ & $<0.001^{* * *}$ \\
\hline $\begin{array}{l}\text { 22. Difficulty with nasal } \\
\text { breathing }\end{array}$ & $3.8 \pm 1.4$ & $1.5 \pm 1.4$ & $<0.001^{* * *}$ \\
\hline 23. Suffocation & $3.4 \pm 1.7$ & $1.5 \pm 1.5$ & $<0.001^{* * *}$ \\
\hline 24. Nose is too open & $1.3 \pm 1.8$ & $0.6 \pm 0.9$ & $0.009^{* *}$ \\
\hline 25. Nasal crusting & $1.0 \pm 1.5$ & $0.9 \pm 1.1$ & 0.514 \\
\hline SNOT-25 total score & $62.9 \pm 25.3$ & $35.5 \pm 24.4$ & $<0.001^{* * *}$ \\
\hline BDI-II score & $19.3 \pm 15.0$ & $8.4 \pm 10.1$ & $<0.001^{* * *}$ \\
\hline BAI score & $17.7 \pm 12.9$ & $10.5 \pm 11.5$ & $<0.001^{* * *}$ \\
\hline
\end{tabular}

Data are represented as mean \pm standard deviation. SNOT-25, sino-nasal outcome test-25; BDI-II, Beck Depression Inventory II; BAI, Beck Anxiety Inventory. † Paired t test analysed between pre-operative and 6 months post-operative symptom scores. ${ }^{*} p<0.05,{ }^{* *} p<0.01,{ }^{* * *} p<0.001$.

Using SNOT-25 metrics to detect moderate-to-severe depression

Given the concern that patients with moderate-to-severe depression (BDI-II score of $\geq 20$ ) warrant immediate psychological intervention and therapy, ROC curves were generated and the AUC was calculated to evaluate if any of the SNOT-25 measures
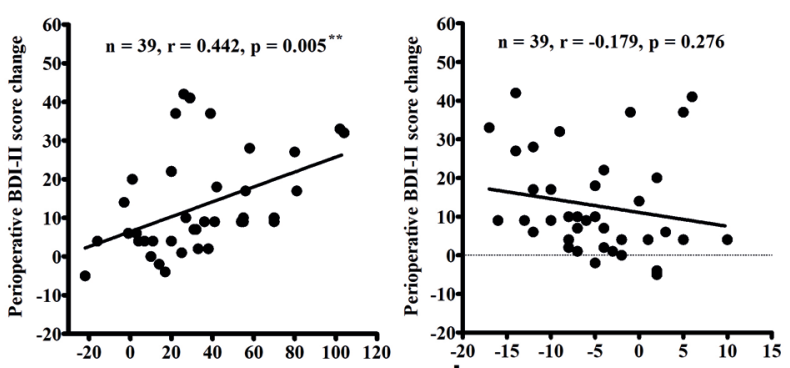

a.

$\begin{array}{lllllll}-20 & -15 & -10 & -5 & 0 & 5 & 10 \\ \text { b. Rhinogenic symptoms change }\end{array}$
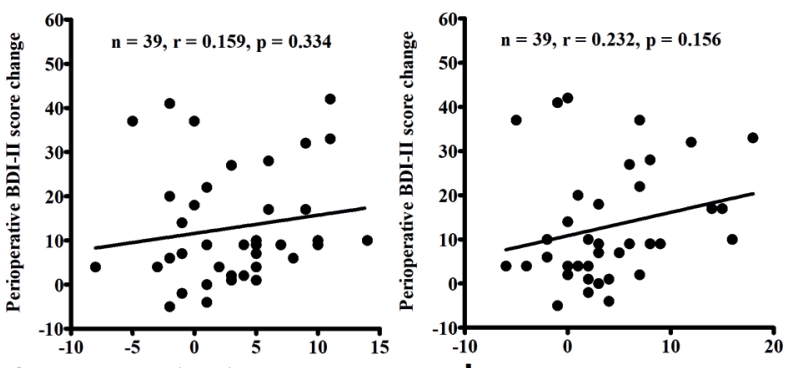

C. Extra-nasal rhinologic symptoms change
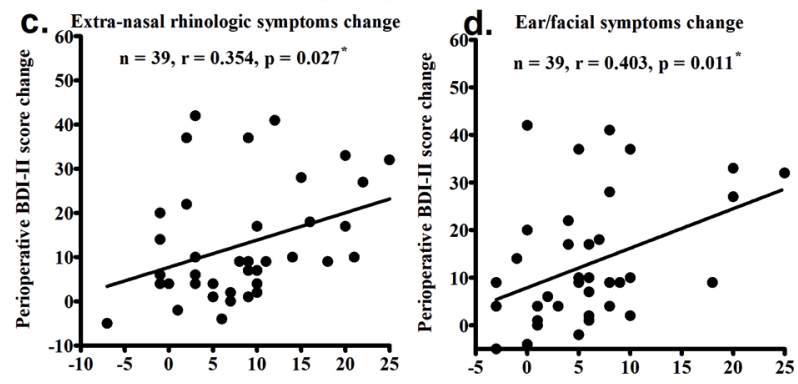

e.

f. ENS symptoms change

Figure 3. Post-operative changes in the Beck Depression Inventory II (BDI-II) score were significantly associated with changes in the Sinonasal Outcome Test-25 (SNOT-25) total score (a), sleep dysfunction domain (e), and empty nose symptoms (ENS) domain (f), but not with the rhinogenic symptoms domain (b), extra-nasal rhinologic symptoms domain (c), or ear/facial symptoms domain (d). The correlation was determined using Pearson's correlation coefficient. ${ }^{*} p<0.05,{ }^{* *} p<0.01$, *** $p<0.001$.

and subdomains (with the exception of the psychological dysfunction domain) would detect moderate-to-severe depression in our participants better than a random test in a statistically significant manner (AUC of $>0.5$ ) (Figure 4). The SNOT-25 total score (AUC $=0.765, p<0.001$ ), sleep dysfunction domain (AUC $=0.734, \mathrm{p}<0.001$ ), and empty nose symptoms domain (AUC = $0.819, p<0.001$ ) all had ROC curves with AUCs that were $>0.5$. The optimal cutoffs for these metrics (maximising the sum of sensitivity and specificity) were a SNOT-25 total score of $>60$ (sensitivity: $84.6 \%$, specificity: $61.9 \%$ ), sleep dysfunction domain score of > 18 (sensitivity: $73.1 \%$, specificity: $66.7 \%$ ), and empty nose symptoms domain score of $>14$ (sensitivity: $75.0 \%$, specificity: $81.6 \%)$. 


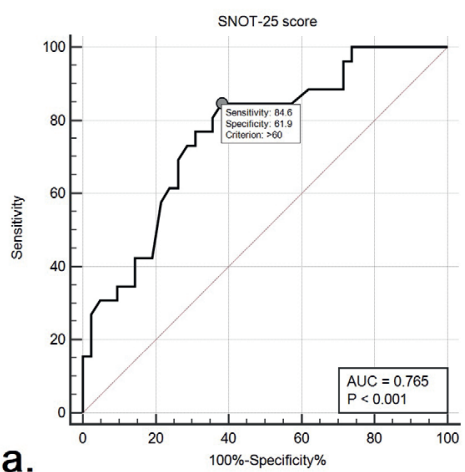

a.

Figure 4. Receiver operating characteristic (ROC) curves to detect moderate-to-severe depression (Beck Depression Inventory II (BDI)-II scores of $\geq$ 20) using the variables of the Sinonasal Outcome Test-25 (SNOT-25) total score (a), sleep dysfunction domain (b), and empty nose symptoms (ENS) domain (c). The optimal cutoffs for these metrics (maximizing the sum of sensitivity and specificity) are indicated.

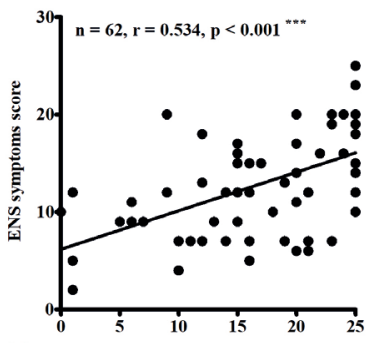

a.

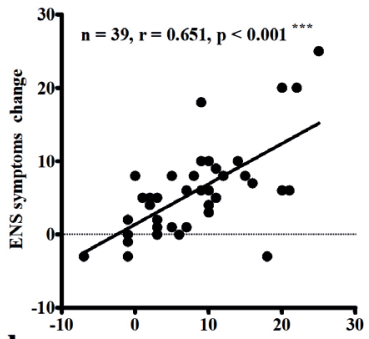

b. Sleep dysfunction change

Figure 5. The sleep dysfunction domain was correlated with the empty nose (ENS) symptoms domain in the baseline (a) and post-operative (b) evaluation.

\section{Discussion}

This is the first study to evaluate the associations of each SNOT25 subdomain with the severity of anxiety and depression in patients with ENS. Previous studies have reported that depression and anxiety are prevalent in ENS patients and could be effectively improved after surgical treatment ${ }^{(8,10)}$. ENS patients carry a substantial psychological burden and experience significant difficulties with many daily activities ${ }^{(8)}$. In the present study, we used validated instruments to evaluate the associations of SNOT-25 and its subdomains with the severity of anxiety and depression before and after surgical treatment in ENS patients. Our results revealed that $38.3 \%$ and $53.0 \%$ of our ENS cohort experienced moderate-to-severe depression and anxiety according to the BDI-II and BAI scores, respectively. The BDI-II and BAI scores were significantly associated with the SNOT-25 total score and ear/facial symptoms, psychological dysfunction, sleep dysfunction, and empty nose symptoms domains. Nasal surgery with Medpor implantation improved both the disease-specific symptoms and the psychological symptoms. Post-operative changes in the BDI-II score were also correlated with changes in the SNOT-25 total score, sleep dysfunction domain, and empty nose symptoms domain. These results indicated that

ENS symptoms, particularly sleep dysfunction and empty nose symptoms, contribute to the psychological burden experienced by ENS patients. Differentiating which disease-specific QOL domains are associated with psychological dysfunction is important and could allow for targeted symptom improvement to reduce the psychological burden.

We next sought to identify patients with moderate-to-severe depression (BDI-II score of $\geq 20$ ) using ROC curve analysis with SNOT-25 metrics and sleep dysfunction and empty nose symptoms domains. ROC curve analysis indicated that a SNOT25 total score of $>60$, sleep dysfunction domain score of $>18$, and empty nose symptoms domain score of $>14$ were good predictors of moderate-to-severe depression. Recognising individuals experiencing heavy psychological burden and providing appropriate intervention are also critical to prevent drastic measures such as suicide attempts in extreme cases.

The link between sleep and depression is strong ${ }^{(17)}$. For example, people with insomnia have greater levels of depression and anxiety than do those who sleep normally. Sleep symptoms cause great distress, have a substantial impact on the QOL and daily activities, and are a risk factor for suicidal ideation ${ }^{(18)}$. In the current study of SNOT-25 subdomain analysis, the sleep dysfunction and empty nose symptoms domains were significantly correlated with the BDI-II and BAI scores, as well as post-operative BDI-II changes. Further analysis revealed a robust association between the sleep dysfunction and empty nose symptoms domains in the baseline evaluation (correlation coefficient $r=$ $0.534, p<0.001$ ), and the post-operative evaluation (correlation coefficient $r=0.651, p<0.001$ ) (Figure 5), although these two subdomains had no overlapping items during the investigation (Table 1). Furthermore, sleep dysfunction, psychological dysfunction, and empty nose symptoms were the most affected domains in the SNOT-25 metrics, with regard to the ratio of the mean score to the full score (Table S). Taken together, it is possible that empty nose symptoms, such as dryness, difficulty 
with nasal breathing, and suffocation, may disrupt the sleep integrity and quality in ENS patients. Poor sleep may have then contributed to or exacerbated the development of anxiety and depression in these patients. As a consequence, from a clinical perspective, acknowledgement and management of sleep problems in ENS patients is one of the most critical issues.

Abnormalities in neurosensory systems due to improper mucosal healing and alterations in nasal airflow after surgical insult are proposed to play major roles in the pathogenesis of $\mathrm{ENS}^{(3-6)}$. Previous studied have demonstrated that inspiration of air directed into a wide nasal passageway lacking inferior turbinates resulted in ineffective air conditioning ${ }^{(4)}$, trigeminal dysfunction ${ }^{(6)}$, and activation of the limbic system ${ }^{(19)}$, and contributed to the ENS symptoms. The surgical treatment by Medpor implantation reconstructed the nasal airway, decreased the width of the passage and volume of the direct cool airflow, and improved the symptoms ${ }^{(13,14,20,21)}$. Hence, the neurosensory abnormality due to improper mucosal healing would not be altered by surgery. Thus, we proposed in this study that the psychological dysfunction may be due to both direct physiological changes in the nasal cavity and indirect interference from the other QOL symptoms, such as sleep dysfunction and empty nose symptoms. This emphasised that collaboration with specialists in psychiatry, psychology, and sleep medicine should serve as adjunctive measures to optimise the treatment outcomes of severe ENS patients.

This study has several limitations that warrant consideration. First, we recruited ENS patients who were receiving surgery. It would have been beneficial to include ENS patients who did not receive surgery or who received sham surgery as a control group in order to exclude the placebo effect. However, this was difficult due to the ethical considerations. Instead, we performed analyses to determine the dynamic correlations between metrics for depression and anxiety and ENS-specific QOL before and after nasal surgery in an ENS cohort. Second, ENS patients who were not undergoing surgery were not enrolled. Patients who opted to receive surgery may have more severe symptoms and QOL impairment than those without surgery. Third, studies of patient-reported measures are susceptible to self-reported biases, such as changes in internal standards, priorities, or the interpretation of a given instrument. Thus, these biases may be present in our findings, although validated instruments (the SNOT-25, BDI-Il, and BAI) were used.

\section{Conclusion}

Symptoms of ENS, particularly the sleep dysfunction and empty nose symptoms domains of the SNOT-25, are associated with psychological burden and could be good predictors for moderate-to-severe depression. Identification of these associations allows for targeted symptom improvement to reduce psychological burden. As patients experiencing heavy psychological burden warrant immediate intervention, this could have a significant impact on the rapid identification and management of such patients.

\section{Acknowledgements}

The authors received a research grant from the Chang Gung Memorial Hospital (CMRPG1H0031, CMRPG3G0672 and CMRPG3G0221). The funder had no role in the study design, data collection, or analysis; decision to publish; or preparation of the manuscript. The authors thank Ms. Meng-Chieh Tsai for aiding the data collection.

\section{Authorship contribution}

TJL and CCH participated in study design. CCH and PWW performed the data collection and analysis, and drafting the manuscript. CCF, CCH, PHC and CLW helped in the enrollment of participants and collection of clinical data. TJL and CCH contributed to the data interpretation. All authors participated in scientific discussion and approved the final manuscript.

\section{Conflict of interest}

The authors declare no conflict of interest.

\section{References}

1. Houser SM. Empty nose syndrome associated with middle turbinate resection. Otolaryngol Head Neck Surg 2006;135:972973.

2. Chhabra N, Houser SM. The diagnosis and management of empty nose syndrome. Otolaryngol Clin North Am 2009;42:311330.

3. Sozansky J, Houser SM. Pathophysiology of empty nose syndrome. Laryngoscope 2015;125:70-74.

4. Zhao K, Blacker K, Luo Y, Bryant B, Jiang J. Perceiving nasal patency through mucosal cooling rather than air temperature or nasal resistance. PLoS One 2011;6:e24618.
5. Chen XB, Leong SC, Lee HP, Chong VF, Wang DY. Aerodynamic effects of inferior turbinate surgery on nasal airflow - a computational fluid dynamics model. Rhinology 2010;48:394-400.

6. Konstantinidis I, Tsakiropoulou E, Chatziavramidis A, Ikonomidis C, Markou K. Intranasal trigeminal function in patients with empty nose syndrome. Laryngoscope 2017;127(6):1263-1267.

7. Jiang C, Wong F, Chen K, Shi R. Assessment of surgical results in patients with empty nose syndrome using the 25 -item sinonasal outcome test evaluation. JAMA Otolaryngol Head Neck Surg 2014;140:453458.
8. Manji J, Nayak JV, Thamboo A. The functional and psychological burden of empty nose syndrome. Int Forum Allergy Rhinol 2018;8(6):707-712

9. Mangin D, Bequignon E, Zerah-Lancner F et al. Investigating hyperventilation syndrome in patients suffering from empty nose syndrome. Laryngoscope 2017;127(9):19831988.

10. Lee $\mathrm{TJ}, \mathrm{Fu} \mathrm{CH}, \mathrm{Wu} \mathrm{CL}$, et al. Evaluation of depression and anxiety in empty nose syndrome after surgical treatment. Laryngoscope 2016;126: 1284-1289.

11. Beck AT, Steer RA, Brown GK. Manual for the Beck Depression Inventory-II. San Antonio, TX: Psychological Corp.; 1996. 
12. Beck AT, Epstein N, Brown G, et al. An inven tory for measuring clinical anxiety: psychometirc properties. J Consult Clin Psychol 1988;56:893-897.

13. Lee $T J, F u C H, W u C L$ et al. Surgical outcome for empty nose syndrome: Impact of implantation site. Laryngoscope 2018;128(3):554-559.

14. Tam YY, Lee TJ, Wu CC, et al. Clinical analysis of submucosal Medpor implantation for empty nose syndrome. Rhinology 2014;52:35-40.

15. Chinese Behavioral Science Corporation. Available at: http://www.mytest. com.tw. Accessed on January 1, 2015.

16. Yeung A1, Howarth S, Chan R, Sonawalla $S$, Nierenberg AA, Fava M. Use of the Chinese version of the Beck Depression Inventory for screening depression in primary care. J Nerv Ment Dis 2002 Feb;190(2):94-9.
17. Nutt D, Wilson S, Paterson L. Sleep disorders as core symptoms of depression. Dialogues Clin Neurosci 2008;10(3):329-36.

18. Thase ME Depression and sleep: pathophysiology and treatment. Dialogues Clin Neurosci 2006:8(2):217-26.

19. Freund W, Wunderlich AP, St€ocker T Schmitz BL, Scheithauer MO. Empty nose syndrome: limbic system activation observed by functional magnetic resonance imaging. Laryngoscope 2011;121:2019-2025.

20. Houser SM. Surgical treatment for empty nose syndrome. Arch Otolaryngol Head Neck Surg 2007;133:858-863.

21. Leong SC. The clinical efficacy of surgical interventions for empty nose syndrome: a systematic review. Laryngoscope 2015;125:1557-1562
Ta-Jen Lee, MD

Division of Rhinology

Department of Otolaryngology

Chang Gung Memorial Hospital and

Chang Gung University,

Taoyuan, Taiwan

No. 5, Fu-Shin Street, Kweishan,

Taoyuan 333, Taiwan

Tel: +886-3-3281200 ext. 8466;

Fax: +886-3-3979361

E-mail: d0300102@stmail.cgu.edu.tw;

entlee@cgmh.org.tw 